\title{
Politique
}

\section{Fortune et infortune de la femme mariée de François de Singly}

\section{Catherine Paradeise}

Numéro 16, automne 1989

Les États-Unis

URI : https://id.erudit.org/iderudit/040637ar

DOI : https://doi.org/10.7202/040637ar

Aller au sommaire du numéro

Éditeur(s)

Société québécoise de science politique

ISSN

0711-608X (imprimé)

1918-6584 (numérique)

Découvrir la revue

Citer cette note

Paradeise, C. (1989). Fortune et infortune de la femme mariée de François de

Singly. Politique, (16), 111-121. https://doi.org/10.7202/040637ar d'utilisation que vous pouvez consulter en ligne.

https://apropos.erudit.org/fr/usagers/politique-dutilisation/ 


\title{
Note critique
}

\section{Fortune et infortune de la femme mariée de François de Singly*}

\author{
Catherine Paradeise \\ Université de Nantes/Lersco
}

Il faut remercier François de Singly, véritable Sherlock Holmes de la sociologie du mariage, pour la rigueur et la clarté avec lesquelles il traque les effets du mariage sur la «fortune» féminine. Ceci à travers une longue investigation empirique qui s'assimile (au sens propre de digestion) une quantité considérable de données statistiques prises à de multiples sources secondaires, enrichies d'enquêtes personnelles, réalisées par ses soins ou sous sa direction.

En première lecture, l'ouvrage de Singly participe de l'entreprise spécifique de désenchantement du monde qui est celle de la sociologie. Il traite en effet du bonheur matrimonial à la façon dont Durkheim traitait du mal de vivre dans Le Suicide. Que

* Paris, PUF, coll. Économie et liberté, 1987, 229 p. 
reste-t-il du mariage si l'on en retranche l'amour? Il reste, nous répond l'auteur, des coûts et des profits sociaux qui peuvent être comptabilisés par le rendement pour chaque conjoint des divers capitaux qu'il apporte en dot dans l'alliance. Cette entreprise d'extraction du social achevée, rien n'interdit cependant de réintroduire l'amour dans le modèle d'analyse, bien au contraire. Non pour analyser classiquement les déterminations sociales de l'affinité élective, mais pour la traiter comme la figure grâce à laquelle se soldent subjectivement les comptes entre les conjoints. Travail qui renvoie aux divers systèmes de représentation de l'amour qui organisent l'alliance et tiennent ensemble les conjoints en leur permettant d'équilibrer leurs coûts et bénéfices respectifs $d u$ point de vue des critères de satisfaction qui leurs sont propres. En effet, les notions de coût et de bénéfice du mariage sont relatives aux représentations spécifiques qui assignent à chaque effet du mariage un signe positif ou négatif.

Ainsi par exemple de la dépendance de la femme: elle sera mise au crédit de son bilan matrimonial, lorsqu'elle s'intégrera dans un système de représentation qui valorise la division sexuelle des rôles et la fusion dans le couple entre l'homme actif et la femme au foyer, mais à son débit lorsqu'elle s'insérera au contraire dans un système de représentation qui valorise l'indifférenciation sexuelle des rôles et l'autonomie des deux conjoints actifs. D'où un ensemble de questions: quel sens donner à l'extension du domaine d'influence du modèle de l'autonomie dans nos sociétés? Quelles tensions cette bipolarité spécifique du mariage entre fusion et autonomie engendre-t-elle? Jusqu'à quel point l'autonomie, qui atteint son expression paroxystique dans le modèle de la cohabitation, laisse-t-elle encore place à l'union conjugale comme processus social producteur de richesse, comme forme stabilisée par l'interdépendance des intérêts entre les conjoints ${ }^{1}$ ?

1. Dans le modèle de la cohabitation, les conjoints entendent en effet équilibrer leurs comptes sans y inclure l'amour. C'est la condition de sa «libération», de son autonomisation comme seul fondement de la co-existence conjugale. Devenu «gratuit», l'amour fait donc l'objet d'une comptabilité propre, dont l'équilibre 
Ces questions, qui peuvent servir de grille de lecture à l'ouvrage, François de Singly les traite essentiellement du point de vue des femmes: que gagnent-elles au mariage en termes de création de richesses personnelles, selon la façon dont elles rentabilisent leurs apports? Mais aussi du point de vue complémentaire des hommes: en quoi bénéficient-ils de l'apport de capitaux féminins dans le mariage? Du couple: y a-t-il création de richesse indivis par le mariage, et si oui, comme se redistribue-t-elle lorsque le couple se dissocie? Des enfants enfin: comment profitent-ils des apports de leurs parents, et en retour, comment restituent-ils ces richesses?

Autant d'évaluations positives ou négatives des comptes donc, que de façon de tenir sa comptabilité! L'image du grand registre des crédits et des dettes sert donc plutôt d'instrument heuristique - en permettant au sociologue de la famille d'éviter les pièges de l'amour comme au sociologue de l'art, la beauté que d'outil d'une comptabilité générale à laquelle l'auteur se refuse. Celle-ci supposerait en effet un critère uniforme de valeur, assurant la commensurabilité et la convertibilité des divers éléments comptables. Mais la métaphore reste puissante, parce que, sans statuer a priori sur les valeurs, elle autorise des comparaisons fouillées entre les composantes de la valorisation directe et indirecte des capitaux masculins et féminins dans le mariage. Pierre Bourdieu nous a familiarisés avec l'usage que F. de Singly fait du terme de capital. Plutôt que d'entrer dans un débat difficile sur les dangers d'un usage mal contrôlé du concept de capital, que souligneront peut-être des critiques économiques de l'ouvrage ${ }^{2}$, retenons plutôt que F. de Singly s'en sert comme d'une désignation commode - parce que synthétique, même si l'on peut penser qu'un autre terme eût été plus approprié ici - pour décrire toute une variêté d'attributs individuels qui se mettent en scène dans le mariage: origine sociale (capital social), sociabilité (capital rela-

justifie à lui seul l'association conjugale et le déséquilibre la rupture. On y reviendra.

2. Voir à ce propos la critique de l'ouvrage par C. Baudelot dans la Revue économique. 
tionnel), apparence physique (capital esthétique), formation scolaire (capital scolaire ou culturel), cette gamme de caractéristiques pouvant être étendue à volonté. Par valorisation directe il faut entendre la création de richesse revenant à la femme par son propre travail; par valorisation indirecte, la création de richesse lui revenant par l'intermédiaire du travail de son conjoint (et de ses enfants), dont les capitaux propres peuvent être bonifiés par l'apport féminin dans le couple.

Le mariage est le lieu d'une transformation des conditions de rentabilisation des divers capitaux apportés par les conjoints, productrice de plus de richesse pour la famille que ne le ferait la somme des valorisations individuelles des ressources de ses membres. Elle est l'hypothèse centrale de l'ouvrage, on l'aura déjà compris. Ce ne sont donc pas tant les caractéristiques du mariage comme produit des attributs sociaux - perspective la plus traditionnelle dans la sous-discipline - que les transformations des attributs sociaux engendrées par le mariage qui intéressent ici F. de Singly.

La seconde hypothèse est que la nature, le montant et la pérennité de ces nouvelles richesses varient selon le montant absolu et relatif des apports de chaque associé, mais aussi selon leur sexe. Cela permet à l'auteur de confirmer le constat de la sociologie féministe de la famille: les capitaux ont un sexe, qui se dévoile (!) au moment de la dissolution de l'union. On constate alors que la préservation des capitaux d'origine et l'appropriation des richesses nouvelles créées par le mariage ne sont pas homogènes de l'homme à la femme.

Le troisième hypothèse pose que les diverses formes de contribution des conjoints à la création de richesses - qui, du côté de la femme, jouent sur toute la gamme des valorisations médiate et immédiate de ses capitaux propres — s'associent à des «codes de conduite conjugale» spécifiques, qui - tendus entre les valeurs d'autonomie et de dépendance - assurent la communication entre les époux sur le sens de l'entreprise matrimoniale, et stabilisent leurs attentes mutuelles en équilibrant leurs comptes respectifs. Le couple apparaît donc comme un lieu de tension dans et entre des valeurs et des pratiques, qui engendre des formes spécifiques de 
dépendance des intérêts de chaque membre du couple à l'égard de son conjoint. La vie conjugale n'est par conséquent ni la juxtaposition de deux vies individuelles, ni une vie caractérisée par l'unité d'intérêts indifférenciés. C'est un espace de jeux de coopération et de conflit dont l'enjeu est constitué par la définition et la défense des valeurs et des intérêts communs. À ce titre, la vie conjugale est justifiable d'une analyse sociologique à part entière.

Ainsi traités, les bénéfices et les coûts du mariage se révèlent ambigus pour la femme (là résident tout à la fois sa fortune et son infortune). En effet, l'alliance tend à restreindre la valorisation immédiate des apports féminins, et à subordonner leur valorisation médiate à sa dépendance. Ces deux propositions doivent évidemment être pondérées selon les niveaux absolus et relatifs des capitaux détenus initialement par chacun des conjoints. Vu du côté de la femme, le prix du mariage - joug précieux ou joug pesant selon ses critères de satisfaction, mais toujours principe d'équilibre de ses comptes - est donc sa dépendance. L'ambiguïté de ce prix est bien marquée dans le coût du renoncement au mariage par l'épouse. La séparation sera «heureuse» ou «malheureuse» selon que les bénéfices de sa nouvelle autonomie compenseront ou non l'ancien prix de sa dépendance. Or, les perspectives de valorisation offertes à la femme séparée varient fortement selon qu'elle a vécu son mariage sur le mode de la fusion - par la valorisation médiate de ses apports pour l'essentiel - ou sur le mode de l'autonomie relative - en associant valorisations immédiate et médiate. Lors de la rupture, le mari s'appropriera en effet l'ensemble des contributions de son exépouse à la valorisation de ses capitaux sur le marché du travail (comme d'un fonds de commerce qu'ils auraient conjointement fait fructifier). Par ailleurs, les perspectives de valorisation immédiate des ressources propres à l'épouse se seront d'autant plus détériorées qu'elle ne les aura pas antérieurement investies sur le marché du travail. On comprend ainsi le ressort sociologique de la revendication d'autonomie de la femme mariée active, et de la revendication de fusion de la femme au foyer, considérées comme des type-idéaux de comportement: tout se passe comme si la première, évaluant les risques de dissolution de l'union, cherchait à préserver 
ses perspectives de valorisation sans parier sur la pérennité de son union; tout se passe comme si la seconde ne pouvait préserver ses richesses qu'en pérennisant l'union, et revendiquait en conséquence l'extension maximale de la sphère commune, condition d'organisation de la dépendance et du contrôle matrimonial, source d'augmentation du «coût de séparation» ou encore instrument de réduction du risque de dissolution du couple. Sans doute est-ce là le sens de la remarque très suggestive de F. de Singly, selon laquelle les difficultés conjugales s'exprimeraient chez la femme au foyer par la dépression - qui utilise le ressort de la dépendance masculine pour préserver son union, en substituant un contrôle moral au contrôle social défaillant - et chez la femme active par le divorce - qui lui permet de clore les comptes sans réduire ses perspectives ultérieures de valorisation. Un réconfort cependant pour l'adepte de la valorisation médiate: en cas de rupture du contrat de mariage, il lui reste la possibilité de reconstruire sa richesse en reconstituant sa dépendance par le re-mariage, puisqu'elle conserve sur le marché matrimonial la valeur qu'elle a conquise par son premier mariage.

La méthode utilisée est parfaitement appropriée à une comptabilité dont la vocation est d'organiser la démarche comparative par laquelle l'auteur confronte les effets du mariage sur une population segmentée simultanément ou alternativement par le sexe, l'activité professionnelle, le diplôme, l'âge, le nombre d'enfants, etc. On admirera ici la véritable virtuosité de François de Singly dans le maniement et la présentation des résultats d'un travail extrêmement minutieux d'analyse multivariée, dont chacun sait combien il peut se révéler fastidieux pour ses victimes ou lecteur... Le choix des données et le mode de cheminement des unes aux autres n'apparaissent jamais gratuits: chaque nouvel indice est finement ciselé pour approfondir, tester, vérifier, reformuler le sens général du propos. Quant à la présentation des résultats, si elle requiert une attention soutenue pour ne pas s'égarer dans les dédales d'une démonstration robuste mais construite par petites touches, elle réalise la performance d'atteindre une lisibilité maximum - moins de 40 tableaux statistiques, tous très tra- 
vaillés et très convaincants, augmentés de quelques annexes sans escamoter aucun des divers moments de la démonstration.

Si l'analyse multivariée donne tout à la fois ossature et contenu à l'ouvrage, elle comporte des limites inhérentes qui invitent peut-être à poursuivre le travail à partir d'autres matériaux. Cela dit avec prudence et sans chercher querelle à l'auteur, dont les enquêtes complémentaires répondent déjà partiellement à ce besoin. L'analyse multivariée, par construction pour ainsi dire, contraint en effet à des hypothèses parfois fortes sur le sens de comportements qu'elle permet d'observer, sans donner d'autre instrument de vérification que la convergence des indices qu'elle élabore. Dans certains cas, cela tient au fait que le sens de la causalité ne peut pas être déduit de l'ordre temporel entre les variables; dans d'autres, cela tient au fait que l'angle de coupe privilégié par la méthode interdit d'analyser suffisamment finement le glissement des attributs pertinents de la personne au cours du cycle de vie. Ainsi le chercheur peut-il brutaliser les processus producteurs du comportement en les réduisant à une causalité située en amont de tel ou tel des moments qui l'engendrent.

Prenons à titre d'exemple la façon dont François de Singly interprète la liaison statistique qu'il observe entre le taux d'activité des femmes avant la naissance de leur premier enfant et le nombre d'enfants finalement engendrés. Il émet l'hypothèse que les femmes «maîtrisent leur temps fertile et leur temps professionnel, consciemment ou non» (p. 94). L'inactivité résulterait donc d'un arbitrage a priori entre perspectives maternelles et professionnelles. C'est une hypothèse ... mais il y en a bien d'autres! On peut en effet imaginer bien des processus sociaux ou psychologiques qui feraient de l'accroissement de la famille une conséquence non anticipée de l'inactivité, de même qu'on peut facilement envisager que la poursuite de l'inactivité puisse être une conséquence non anticipée d'un choix temporaire d'interruption du travail au moment de la naissance du premier enfant. Les comportements peuvent avoir une régularité dans leurs effets sans qu'il faille nécessairement présupposer que les acteurs maîtrisent leur principe d'efficacité. F. de Singly le sent bien, puisqu'il désigne ce principe du terme embarrassé, et pour le moins paradoxal, 
de «maîtrise inconsciente»! Mais il faudrait pourtant trancher entre maîtrise et inconscience (qui sont d'ailleurs sans doute inégalement partagées selon les milieux sociaux et les moments historiques). En effet, on bien la relation construite par l'analyse multivariée exprime sans médiation le fondement de l'action des femmes: dans ce cas, elles maîtrisent effectivement leur temps professionnel et leur temps fertile, c'est-à-dire qu'elles renoncent au moins partiellement à l'un des termes de l'alternative en faveur de l'autre. Ou bien, on doit chercher ailleurs - ou autrement - que dans cette relation les principes qui la produisent, et l'hypothèse de l'inconscience des enchainements des causes et des effets est recevable.

$\mathrm{Si}$, dans le contexte de la méthode, l'interprétation de l'auteur s'impose comme la plus plausible, parce que la plus cohérente et la plus économique, il ne faut pourtant pas oublier que la vision en coupe qui la produit interdit de saisir la reconversion des attitudes et des opportunités lorsque se modifie la configuration de vie des femmes et des couples. Ainsi les processus irréversibles engendrés par une suite de micro-décisions risquent-ils d'être figés par la méthode en stratégies d'acteurs, comme si chaque «coup» joué n'affectait pas les coups jouables ultérieurement. Cet aspect des choses est pourtant décisif, tant en matière de choix matrimoniaux et de fécondité qu'en matière de choix professionnels: de même que la temporalité asexuée des rythmes d'acquisition scolaire affecte les opportunités des femmes sur un marché matrimonial à chronologie sexuée, de même la temporalité asexuée construite par la structure du marché du travail - fondée sur la précocité et la continuité de la carrière - affecte leurs opportunités de valorisation en cas de reprise d'activité professionnelle. Si le mariage produit des effets sexués sur la valorisation des capitaux des conjoints, n'est-ce pas que la femme est, bien plus que l'homme, inscrite dans des rythmes biologiques et sociaux décalés par rapport à ceux de la production? Plutôt que de temporalité asexuée, il vaudrait mieux alors parler de temporalité construite par, et ajustée aux rythmes de la vie matrimoniale et professionnelle des hommes avant que n'interviennent la transformation des chances de vie des femmes, qui s'impose socialement aux femmes lorsqu'elles 
assument des rôles traditionnellement dévolus aux hommes. De ce fait, les femmes - qu'elles en soient ou non conscientes, et la conscience ne fait ici rien à l'affaire - sont plus exposées aux conséquences de micro-décisions qui représentent objectivement des options entre les termes de multiples alternatives, parce qu'elles entraînent des effets irréversibles dans la vie matrimoniale et la vie professionnelle, produits par la structure actuelle du marché matrimonial et du marché du travail: celle qui n'a pas «choisi» le marché du travail au moment «voulu» (c'est-à-dire normé par la structure des filières de carrière professionnelles) a de fait largement réduit ses chances dans tout un ensemble de «carrières»; inversement, celle qui n'a pas «choisi» le mariage au moment «voulu» (c'est-à-dire normé par la structure du marché matrimonial) a de fait largement réduit ses chances de vie familiale. Le renoncement «objectif» à la carrière professionnelle n'engendrait aucun problème subjectif pour les femmes aussi longtemps que leur «non-travail» constituait une norme sociale puissante, associée à des perspectives stables de valorisation indirecte par le mariage; ce n'est plus le cas à partir du moment où le développement de la scolarisation contamine l'univers féminin avec ses valeurs "asexuées», lorsque le marché du travail offre aux jeunes filles des opportunités de valorisation directe de leurs titres, lorsque la transformation des modes de vie rendent les perspectives de valorisation indirecte plus incertaines, sans qu'on puisse dans tout cela démêler simplement l'écheveau des effets et des causes. La transformation rapide des chances de vie féminines associées aux perturbations de l'insertion des femmes dans l'ordre matrimonial, scolaire, professionnel, explique ainsi que l'innovation en matière matrimoniale soit d'abord venue des femmes, les hommes y étant entraînés en quelque sorte par ricochet. Non sans qu'ils puissent - comme l'ont souligné de nombreux essais féministes - jouir au passage, provisoirement sans doute, d'une «rente de modèle culturel», en réinterprétant l'innovation à leur bénéfice dans les termes du double standard traditionnel.

L'ouvrage de F. de Singly foisonne de résultats auxquels il est impossible de rendre justice dans un bref compte-rendu. Revenons simplement pour conclure sur la question très actuelle de la 
cohabitation comme processus de désualisation des rôles des conjoints relativement au travail, au pouvoir, aux charges domestiques, à l'accès aux ressources culturelles, associé à l'expression et à la pratique exacerbées de l'autonomie. Le modèle d'interprétation que nous offre l'auteur permet de comprendre, dans la sphère matrimoniale, les processus d'individuation caractéristiques de nos sociétés. En effet, dans les termes de la comptabilité des conjoints, la cohabitation apparaît comme un mode de réduction de l'écart croissant entre les apports et les rétributions des femmes, à mesure que le pari de la valorisation indirecte devient plus risquée - c'està-dire que la probabilité de dissolution du couple s'accroît - et à mesure que les perspectives de valorisation directe s'améliorent. Sa contrepartie est la restriction de la zone commune des conjoints, de ce qui constitue à proprement parler la famille comme lieu de multiplication de richesses. Le «mariage sans frais» (selon l'expression de François de Singly), condition de la rupture sans douleur (sinon morale), ne laisse - au moins est-ce l'hypothèse de l'auteur - presque plus d'espace propice à la fructification commune. En d'autres termes, en cherchant à résorber la tension entre autonomie et fusion propre au mariage d'amour dans les sociétés contemporaines, la cohabitation dissout les effets propres du mariage. Curieuse coöncidence entre l'utopie d'une société où les progrès de la biologie permettraient à la production de la vie d'échapper à la famille - qui perdrait ainsi l'un de ses ciments les plus puissants - et l'utopie d'un amour détaché des intérêts sociaux qui consolident l'alliance. La société achèverait ainsi, dans une «sociologie-fiction», un cycle qui l'aurait conduite du mariage sans amour à l'amour sans mariage. L'alliance ne serait plus «l'angoissante aventure» sociale qu'en faisait Claude Lévi-Strauss, puisqu'elle n'engagerait plus cette opération spécifique de transformation et de multiplication des richesses qui caractérise la famille telle que nous la connaissons. La cohabitation achèverait ainsi paradoxalement le modèle du mariage d'amour, en privant de sens le contrôle social qui encadrait l'alliance et stabilisait l'institution familiale. Pour revenir à une science non fictive, il suffira pourtant de faire état de toute la distance qui sépare l'utopie de la cohabitation de sa réalité, et qui, en dépit de l'accentuation du 
pôle de l'autonomie dans le couple, préserve - sous des formes que seule la recherche empirique permettra de préciser - le pôle de la fusion. 